\title{
Reduction of ice adhesion using Surface Acoustic Waves: nanoscale vibration and interface heating effects
}

XingChang Zeng ${ }^{a, b}$, ZeXiang Yan ${ }^{a}$, YuChao Lu ${ }^{a}$, YongQing Fu ${ }^{c}$, XiangLian Lv ${ }^{a}$, WeiZheng Yuan ${ }^{a}$, Yang He ${ }^{* a}$ a Key Laboratory of Micro/Nano Systems for Aerospace, Ministry of Education and Shaanxi Key Laboratory of Micro and Nano Electromechanical Systems, School of Mechanical Engineering, Northwestern Polytechnical University, Xi'an 710072, P. R. China

b Xi'an institute of applied optics, Xi'an 710072, P.R.China

c Faculty of Engineering \& Environment, Northumbria University, Newcastle upon Tyne, NE1 8ST, UK

\section{Section 1: Characterization of SAW device surface}

The SAW device surfaces (which were coated with a layer of CY Top to make it hydrophobic) were tested with a contact angle analyzer (OCA15EC, Germany, Data Physics) to characterize the static water contact angles and contact angle hysteresis. The test results show that the average values of the static contact angle, advancing angle and receding angle of SAW surfaces were $\sim 102^{\circ}, \sim 115.7^{\circ}$ and $\sim 75.3^{\circ}$, respectively, implying the contact angle hysteresis of around $40.6^{\circ}$. The results show that the SAW device surface is hydrophobic with a strong water adhesion and the droplet is in a Cassie-Wenzel state on the surface as shown in FIG. S1. 


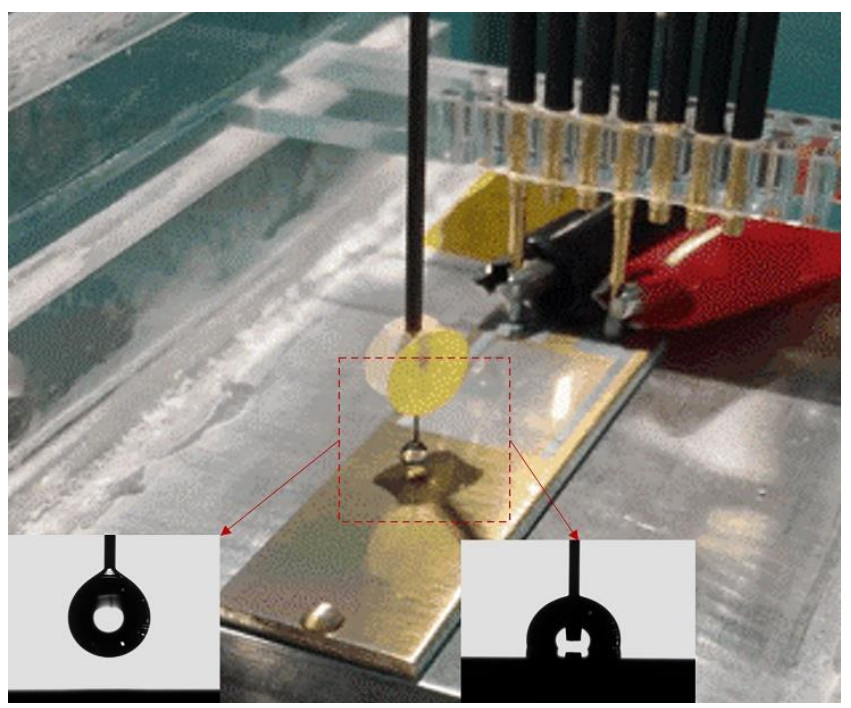

FIG.S1. Characterization of SAW surface wettability

\section{Section 2: Theoretical derivation}

The electrostatic force calculated by Coulomb force equation: The surface is assumed to be smooth, and the electrostatic force, e.g., the main force of the adhesion strength between the ice and the substrate, is calculated using the Coulomb force equation given by:

$$
F_{\text {es-water }}=\frac{q_{\text {es-water }} q_{e s-\text { substrate }}}{4 \pi \varepsilon_{0} \varepsilon_{\text {water }} \delta_{\text {water }}^{2}},
$$

where $F_{\text {es-water }}$ denotes the electrostatic force of the droplet on the surface of substrate, It is further assumed that the total charge of droplet contact surface $q_{e s-w a t e r}$, is equal to the total charge of substrate contact surface $q_{\text {es-substrate }}$ as the water droplet freezes to ice on the surface. $\delta_{\text {water }}$ is the average distance between the droplet and the substrate interface, $\varepsilon_{0}$ is the dielectric constant of the base material, $\varepsilon_{\text {water }}$ is the dielectric constant of deionized water.

Calculation of adhesion work on the SAW surface before droplet frozen into ice: The work of adhesion $W_{a}$, between the droplet and the SAW surface can be defined as:

$$
W_{a}=\gamma_{S G}+\gamma_{L G}-\gamma_{S L}=\frac{W_{A}}{A_{\text {water }}}
$$


where $\gamma_{S G}, \gamma_{L G}, \gamma_{S L}$ are the surface tensions at the substrate/ vapor, water/ vapor, water/ substrate interfaces, respectively. The surface tension is expressed as a force per unit length, which also represents energy per unit area. According the Young equation, the surface tensions are related in the following equation:

$$
\gamma_{S G}=\gamma_{S L}+\gamma_{L G} \cos \theta
$$

$\cos \theta$ is the cosine of static contact angle of liquid.

Calculation relationship of electrostatic force and adhesion work: The total work of adhesion can be expressed as:

$$
W_{a}=\frac{W_{A}}{A_{\text {water }}}=\frac{\int_{0}^{\delta_{\text {water }}} F_{e s-\text { water }} d x=F_{\text {es-water }} \delta_{\text {water }}}{A_{\text {water }}}
$$

As $A_{\text {water }}$ is unit area:

$$
W_{a}=\frac{F_{\text {es-water }} \delta_{\text {water }}}{A_{\text {water }}}=\gamma_{L G}(1+\cos \theta)
$$

Combining Eq.(S2) to Eq. (S6), the adhesion strength of the liquid on the smooth surface is given by:

$$
\tau_{\text {adh-water }}=\frac{F_{e s-\text { water }}}{A_{\text {water }}}=\frac{\gamma_{L G}}{\delta_{\text {water }}}[1+\cos \theta]
$$

where $A_{\text {water }}$ the contact area between the liquid and the substrate surface. $\theta$ is water static contact angle.

For a rough surface, the relationship between the water contact angle and macroscopic roughness or Root Mean Square (RMS) roughness can be described as:

$$
\frac{\theta}{\theta_{c-R M S}}=f(k)
$$

Therefore, the water adhesion for a rough surface is

$$
\tau_{a d h}=\frac{\gamma_{L G}}{\delta_{\text {water }}}\left[1+\cos \left(\theta_{C-R M S} f(k)\right)\right]
$$

Assuming the ice adhesion or shear stress is proportional to the water adhesion or shear stress, and also taking into account the change in electrostatic properties, the ice adhesion can be written as: 


$$
\tau_{\text {adh-ice }}=\alpha_{i c e} \frac{\gamma_{L G}}{\delta_{i c e}}\left[1+\cos \left(\theta_{C-R M S} f(k)\right)\right]
$$

where $\alpha_{i c e}$ is compensation coefficient for the difference in electrostatic force between water and ice, $\delta_{i c e}$ is the average distance between the ice and the substrate interface.

If we assume that the droplet is in the Cassie state, after solidification, the ice adhesion can be divided into two parts: (1) the electrostatic force between the ice in contact with the substrate at a molecular distance $\delta_{i c e}$, which is similar to removing the ice from the substrate roughness element top; and (2) the electrostatic force between the ice and the ice covering the hollow which is not in contact with the substrate but at a distance $k$ corresponding to the roughness height. Based on this assumption, the ice adhesion can be expressed for a rough substrate is

$$
\tau_{\text {adh-ice }}=\alpha_{i c e} \frac{4 \gamma_{L V}}{\delta_{i c e}} f_{R M S}+\alpha_{i c e} \frac{4 \gamma_{L V}}{k}\left(1-f_{R M S}\right),
$$

where the $f_{R M S}$ is the contact rate of ice with the substrate surface and $k$ is the Root Mean Square roughness height.

$$
\tau_{a d h-i c e}=\alpha_{i c e} \frac{\gamma_{L G}}{\delta_{i c e}}\left[1+\cos \left(\theta_{C-R M S} f(k)\right)\right] f_{R M S}+\alpha_{i c e} \frac{\gamma_{L G}}{k}\left[1+\cos \left(\theta_{C-R M S} f(k)\right)\right]\left(1-f_{R M S}\right)
$$
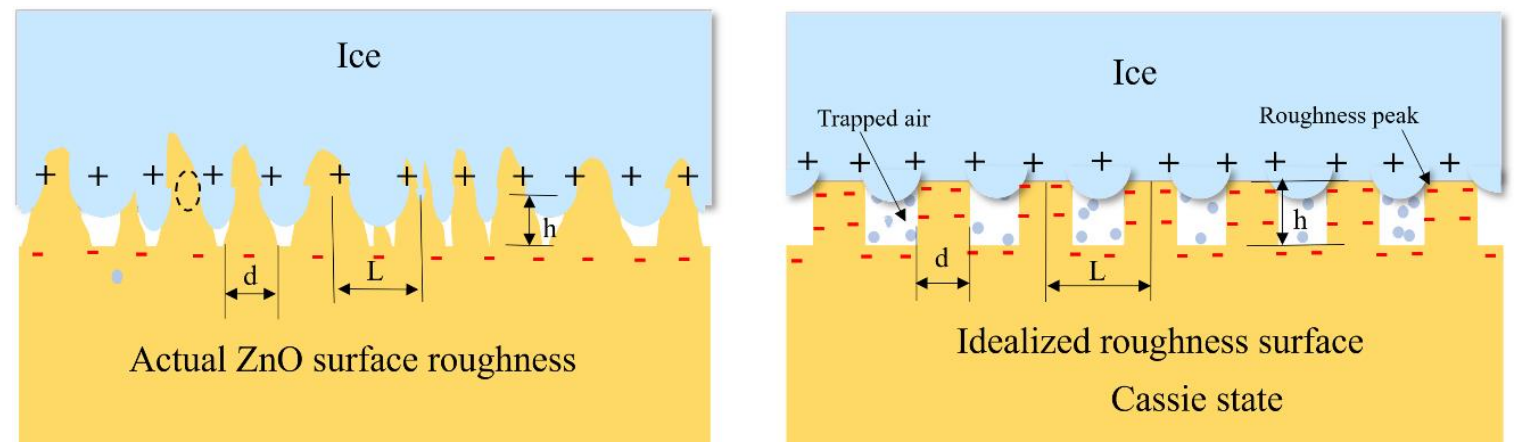

FIG.S2 Idealized Characterization of SAW Surface Topography

Physical Parameters: In the following we introduce some useful parameters which we used in the Eq.(S10)(S11) about surface shown in FIG. S2. $k$ is the root mean square roughness, which corresponds to the roughness height: 


$$
\mathrm{k}=h_{R M S}, h_{R S M}=\sqrt{\frac{1}{n} \sum_{i=1}^{n}\left(h\left(x_{i}\right)-\bar{h}\right)^{2}}, \bar{h}=\frac{1}{n} \sum_{i=1}^{n} h\left(x_{i}\right),
$$

where $n$ is the number of lattice points, $h\left(x_{i}\right)$ the height at lattice site $x_{i} . \bar{h}$ is the average height. $f_{R M S}$ is the fraction of ice in contact with the substrate as defined by formula:

$$
f_{R M S}=\frac{L_{R M S}^{2}}{d_{R M S}^{2}}
$$

where $L_{R M S}$ is the RMS roughness width, $d_{R M S}$ is the RMS roughness distance. The roughness distance and the roughness height can be represented similarly. The RMS roughness distance is defined as

$$
\mathrm{d}_{\mathrm{RMS}}=\sqrt{\frac{1}{\mathrm{n}} \sum_{i=1}^{n}\left(d\left(x_{i}\right)-\bar{d}\right)^{2}}, \quad \bar{d}=\frac{1}{n} \sum_{i=1}^{n} d\left(x_{i}\right),
$$

where $d\left(x_{i}\right)$ is the distance between adjacent roughness elements at lattice site $x_{i}$. The RMS roughness width is defined as:

$$
\mathrm{L}_{\mathrm{RMS}}=\sqrt{\frac{1}{\mathrm{n}} \sum_{i=1}^{n}\left(L\left(x_{i}\right)-\bar{L}\right)^{2}}, \quad \bar{L}=\frac{1}{n} \sum_{i=1}^{n} L\left(x_{i}\right)
$$

where $L\left(x_{i}\right)$ is the roughness of flathead at lattice site $x_{i}$.

Calculation of ice contact area : In Section 1, we have already known that the average value of the static water contact angle is $102^{\circ}$ on the SAW device surface. Thus, the contact area of the ice can be considered as a circular cross section of the sphere as shown in FIG.S3a.
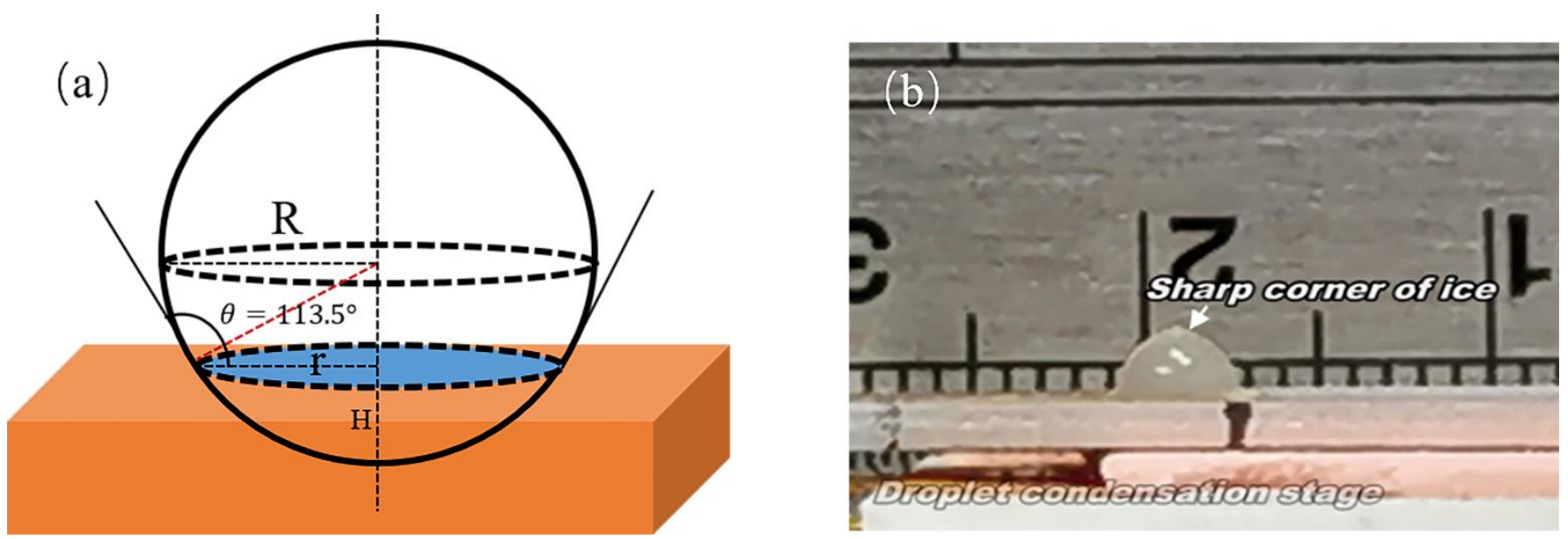

FIG.S3 Ice contact with the surface (a) schematic diagram of shape of ice frozen on surface;(b) The Photo of the ice frozen on surface. 
The radius (r) of ice contact area can be calculated by the following relation:

$$
\begin{aligned}
& V=\frac{4}{3} \pi R^{3}-\frac{1}{3} \pi(3 R-H) H^{2} \\
& H=R\left[1-\sin \left(\theta-90^{\circ}\right)\right] \\
& r=R \times \cos \left(\theta-90^{\circ}\right)
\end{aligned}
$$

where $V$ is the volume of the ice frozen from $25 \mu L$ droplet, thus $V$ is approximately $25 \mathrm{~mm}^{3}, R$ is the radius of ice sphere, $\theta$ is the static water contact angle, $H$ is the height of the sphere being interception, $r$ is the radius of ice contact area. Based on the above formulas, we can calculate the radius of ice contact area $r=$

\section{$2.397704 \mathrm{~mm}$.}

The actual measured radius $\mathrm{r}=2 \mathrm{~mm}$, as shown in FIG.S3b. Since the droplet was micro-sized $(25 \mu L)$, the specific surface area was high, therefore evaporation during experiments may have an impact on the reduction of the droplet volume that may cause the difference between the calculated radius and the measured ones. To avoid the evaporation influence, the measured radius was selected to calculate the contact area, which was $s=\pi r^{2}=12.56 \mathrm{~mm}^{2}$.

Calculation of average dipole spacing: The SAW applied on the surface of $\mathrm{ZnO}$ piezoelectric substrate will cause the nano-vibration of the interface particles, which will cause the average dipole spacing $\delta_{\text {ice }}$ of the electrostatic interaction between the ice and the interface to change. The migration of dielectric atoms during SAW propagation on the surface of $\mathrm{ZnO}$ piezoelectric substrate is given by the following relation:

$$
u_{i}=A_{i} \exp \left\{j\left[\omega t-\beta\left(l_{1} x_{1}+l_{2} x_{2}+l_{3} x_{3}\right)\right]\right\},
$$

where $u_{i}$ is the vibrational displacement of the atom that is the particle at a distance $\mathrm{x}_{1}$ from the sound source in the $\mathrm{i}(\mathrm{i}=1,2,3)$ direction. $l_{1}, l_{2}$ and $l_{3}$ are the cosine of the direction of surface acoustic wave propagation, $x_{1}, x_{2}$ and $x_{3}$ are the propagation distance of the surface acoustic wave along the horizontal, the vertical direction and the normal of plane, $\omega=2 \pi \mathrm{f}$ is the angular frequency, $\beta=2 \pi / \lambda$ is the wave number, $\lambda$ is the wavelength, $\alpha_{i}$ is the amplitude of the wave. $u_{i}$ is the vibration displacement of the atom that is the relative position $\left(x_{1}, x_{2}, x_{3}\right)$ from the sound source, where $x_{3}=0, x_{2}=0$. Assuming that the contact surface 
between ice particles and the interface is circle with a radius of $r_{0}$, and the distance between the center of the circle and the sound source (the end of the interdigital electrode) is $x_{1}$. The average distance change in the surface caused by the surface acoustic wave, can be expressed:

$$
\bar{u}=\frac{A_{i} \int_{0}^{\pi} 2 r_{0} \sin \alpha \exp \left\{j\left[\omega t-\beta l_{1}\left(x_{1}+r_{0} \cos \alpha\right)\right]\right\} d \alpha}{\pi r_{0}^{2}},
$$

where $\alpha$ is the angle between the direction of SAW propagation and the radius of the circle projected by the ice particles on the surface.

\section{Section 3: Thermal effect of SAW surface}

The thermal effect of SAW surface was evaluated by infrared thermal imaging technique at a low temperature $\left(-10^{\circ} \mathrm{C}\right)$ environment. In order to obtain the thermal distribution of SAW surfaces with an input power of $4 \mathrm{~W}$, the temperature change on the SAW device was monitored using an infrared thermal camera (Ti450, FLUKE). The obtained equilibrated temperature distribution of SAW is shown in FIG. S4(a), in which curves L1 and L2 represent the temperature distribution of SAWs. The surface temperature of the device shows a clear gradient distribution from the end of the interdigital transducer to the edge of the device. At the edge of the device, the surface temperature of the device shows a significant increase. The reason is that the intensity of SAW will continue to decay during propagation process with the acousto-thermal effect occurring, and the edge accumulation of SAW energy brings a sharp increase in the acoustic energy density. At room temperature environment, it is verified that the heat generated by the SAW on the surface is quickly absorbed by the cold plate.

As shown in picture FIG.S4(b), at room temperature $\left(23^{\circ} \mathrm{C}\right)$, surface temperature of the SAW device was $23.4^{\circ} \mathrm{C}$. When an excitation of SAW was input, the temperature of SAW surface was rise to $34.7^{\circ} \mathrm{C}$ and then steadily maintained at $34.7^{\circ} \mathrm{C}$. However, As the semiconductor-based Peltier device began to work, the 
temperature of the SAW surface was quickly decreased to $18^{\circ} \mathrm{C}$ and kept at this temperature. The surface temperature started to rise rapidly at 5 s and reached an equilibrate state within 8 s (red line in Figure $S 4(b)$ ). After the semiconductor-based Peltier device was started, the surface temperature was rapidly decreased and reached a thermal equilibrium state within $5 \mathrm{~s}$ (blue line in Figure S4(b)). Results showed that under a certain power, the heat generated by the SAWs wave would make the SAW device surface reaching a thermal equilibrium state within $10 \mathrm{~s}$. Therefore, the time of SAW duration in all ice adhesion strength tests were set at $10 \mathrm{~s}$ in order to exclude the impact of SAW duration.
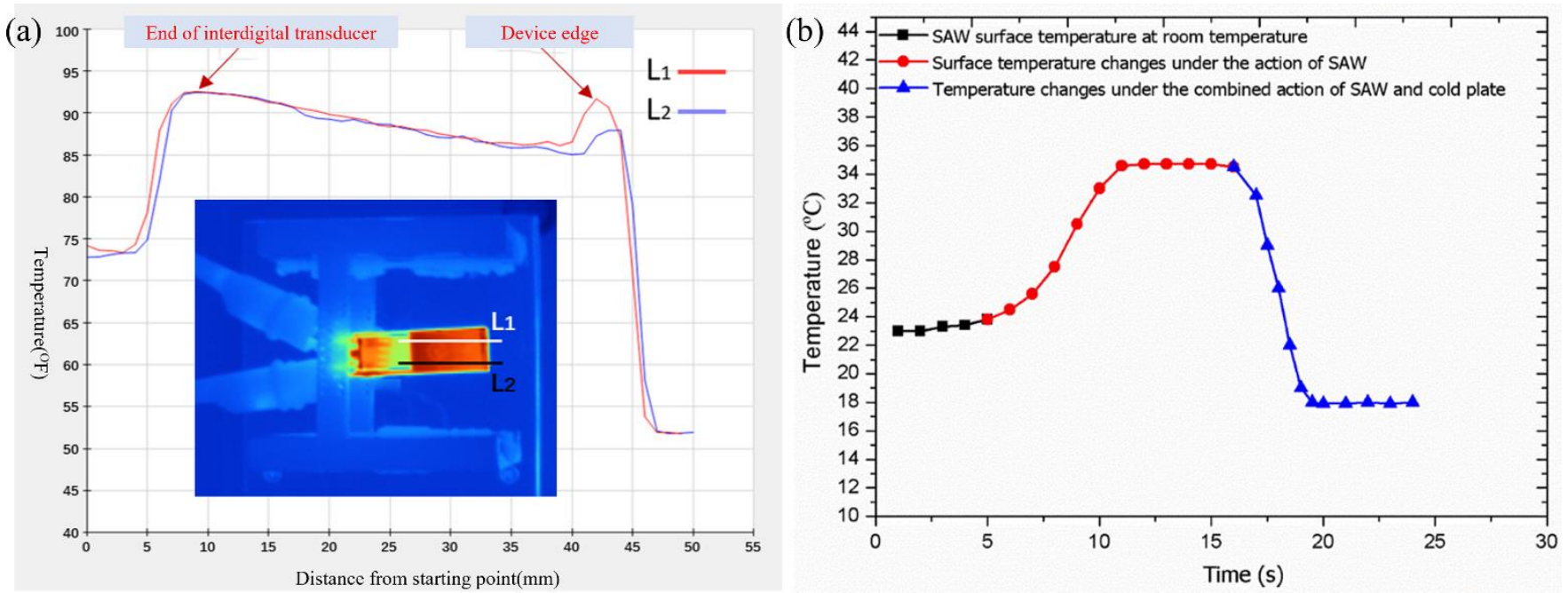

FIG.S4. (a)Thermal distribution of SAW surfaces with input power of $4 \mathrm{~W}$. L1 and L2 represent the temperature distribution of SAW from proximal to distal. (b) the temperature change of SAW device surface under the action of SAW and cold plate. 